\title{
A TGAS/Gaia DR1 parallactic distance to the $\sigma$ Orionis cluster
}

\author{
José A. Caballero ${ }^{1,2, \star}$ \\ 1 Landessternwarte Königstuhl, Zentrum für Astronomie der Universität Heidelberg, Königstuhl 17, D-69117 Heidelberg, \\ Germany \\ 2 Centro de Astrobiología (CSIC-INTA), ESAC campus, Camino Bajo del Castillo s/n, E-28692 Villanueva de la Cañada, \\ Madrid, Spain
}

Received 2016 Nov 19, accepted 2017 February 20

Published online 2017 April dd

Key words stars: distances - stars: early-type - stars: pre-main sequence - Galaxy: open clusters and associations: individual: $\sigma$ Orionis

\begin{abstract}
With the new Tycho-Gaia Astrometric Solution, I derive a new parallactic distance to the young $\sigma$ Orionis open cluster, which is a cornerstone region for studying the formation and evolution of stars and substellar objects from tens of solar masses to a few Jupiter masses. I started with the list of the 46 brightest cluster stars of Caballero (2007). After identifying the 24 TGAS stars in the 30 arcmin-radius survey area and accounting for 11 FGKM-type dwarfs and giants in the fore- and background, I got a list of 13 cluster members and candidates with new parallaxes. Of them, I discarded five cluster member candidates with questionable features of youth and/or discordant parallaxes and proper motions, including a distant Herbig $\mathrm{Ae} / \mathrm{Be}$ star, and proceeded with the remaining eight stars. The $\sigma$ Orionis mean heliocentric distance is $d=360_{-35}^{+44} \mathrm{pc}$, which is consistent with a contemporaneous interferometric determination that concludes a two-decade dispute on the cluster distance. As a by-product, the re-classification of those five cluster member candidates, now interlopers, shows a manifest deficit of cluster stars between 1.2 and $2.1 M_{\odot}$, which leaves the door open to new astrometric membership analyses with the next Gaia data releases.
\end{abstract}

Copyright line will be provided by the publisher

\section{Introduction}

The $\sigma$ Orionis cluster (Garrison 1967; Wolk 1996; Sherry et al. 2004; Walter et al. 2008; Caballero 2008c) is located in the easternmost part of the Ori OB1b association and is one of the most attractive and visited regions for night sky observers. The cluster gets the name from the massive star system in its centre, which is the fourth brightest star in the Orion Belt and visible with naked eye. The $\sigma$ Orionis cluster is important for several reasons:

- Its stars illuminate the Horsehead Nebula photodissociation region (Pound et al. 2003; Pety et al. 2005, 2012; Goicoechea et al. 2006).

- It contains an abundant X-ray emitter population (Mokler \& Stelzer 2002; Franciosini et al. 2006; Skinner et al. 2008; Caballero et al. 2009, 2010).

- It is a cornerstone for studying discs and their frequency at an age of about $3 \mathrm{Ma}$ and at all mass domains (Oliveira et al. 2004; Caballero et al. 2007; Zapatero Osorio et al. 2007; Hernández et al. 2007, 2014; Luhman et al. 2008; Williams et al. 2013; Maucó et al. 2016).

- The prototypical, helium-rich, magnetically active, B2 Vp star $\sigma$ OriE is in its centre (Walborn 1974; Groote \& Hunger 1982; Townsend et al. 2005, 2010; Oksala et al. 2012; Caballero et al. 2016).

^ caballero@cab.inta-csic.es
- It holds four Herbig-Haro objects and dozens Herbig $\mathrm{Ae} / \mathrm{Be}$ and T Tauri stars and brown dwarfs (Haro \& Moreno 1953; Reipurth et al. 1998; Andrews et al. 2004; Caballero et al. 2006; Rigliaco et al. 2011b; Riaz et al. 2015).

- The central trapezium-like star system contains the most massive "binary" with an astrometric orbit (Hartkopf et al. 1996; Mason et al. 1998; Caballero 2008b; SimónDíaz et al. 2011, 2015; Schaefer et al. 2016).

- Its proximity, youth, and low extinction facilitate studies of photometric variability, accretion rates, and accretion frequency at low and very low masses (Zapatero Osorio et al. 2002; Caballero et al. 2004; Scholz \& Eislöffel 2004; Kenyon et al. 2005; Gatti et al. 2008; Sacco et al. 2008; Scholz et al. 2009; Cody \& Hillenbrand 2010; Rigliaco et al. 2011a).

- It is the star-forming region with the largest number of confirmed brown dwarfs $\left(\mathcal{M} \lesssim 72 M_{\text {Jup }}\right)$ and objects below the deuterium burning mass limit (i.e., isolated planetary-mass objects; $\mathcal{M} \lesssim 13 M_{\text {Jup }}$ ) with spectroscopy and youth features (Béjar et al. 1999, 2011; Zapatero Osorio et al. 2000; Caballero et al. 2007, 2012; Bihain et al. 2009; Lodieu et al. 2009; Rigliaco et al. 2012; Peña-Ramírez et al. 2012).

The abundance of known substellar objects has led $\sigma$ Orionis to become a key region all over the sky to study the initial mass function, especially at very low masses. 
However, one of the main caveats in the initial mass function determination in open clusters in general, and in $\sigma$ Orionis in particular, is the uncertainty in heliocentric distance. Its contribution to the final errors is comparable to the uncertainty in age, and far larger than those in theoretical models, contamination rate, or multiplicity (Caballero 2011). Similarly, a more precise and accurate distance to $\sigma$ Orionis would help in improving the determination of disc lifetimes, X-ray luminosities, and the three-dimensional structure of the Ori OB1b association, among other parameters.

There have been different determinations of the distance to the Ori OB1b association and $\sigma$ Orionis cluster, which vary between $352_{-168}^{+166} \mathrm{pc}$ and $473 \pm 33 \mathrm{pc}$ (see below). The closer the cluster, the smaller the distance modulus and the lower the member luminosities. A change of $25 \%$ in the assumed distance can have a significant effect on the derived individual masses, star-to-brown dwarf ratio, or slope and minimum-mass cut-off of the initial mass function, just to cite a few examples (Lodieu et al. 2009).

With the aim of improving previous determinations and of comparing with the precise, contemporaneous, interferometric determination of a distance by Schaefer et al. (2016), I take advantage of the recent release of the Tycho-Gaia Astrometric Solution (TGAS; Gaia Collaboration et al. 2016) for narrowing down a new parallactic distance to $\sigma$ Orionis.

\section{Analysis and results}

First, I retrieved the list of the 46 brightest stars in the $\sigma$ Orionis cluster compiled by Caballero (2007). They are the 41 stars from the Tycho-2 catalogue (TYC2; Høg et al. 2000) within a 30 arcmin-radius area centred on the $\sigma$ Ori Aa,Ab,B triple star (Simón-Díaz et al. 2015), plus the remaining four Tycho-1 (TYC1; Høg et al. 1998) stars in the area not tabulated by $\mathrm{TYC} 2$, and $\sigma$ Ori $\mathrm{C}$, which was skipped by Hipparcos in spite of its relative brightness $(V \approx$ $8.8 \mathrm{mag}$ ) and which proper motion was taken from the NOMAD catalogue (Zacharias et al. 2004). The radius choice was a posteriori ratified by Caballero (2008a), who determined that the $\sigma$ Orionis cluster is composed by a dense core that extends from the centre to $\sim 20$ arcmin and a rarified halo at larger separations. At more than 30 arcmin from the centre, the contamination by neighbouring young populations at similar heliocentric distances gets significant: the younger Alnitak and Horesehead Nebula populations to the north-east and east (Caballero 2008a), the outskirts of the Orion Nebula Cluster to the south and south-west, and the slightly older cluster around Alnilam to the north-west (Caballero \& Solano 2008; Kubiak et al. 2016). The 46 bright stars are sorted in Table A1 by angular separation to the cluster centre.

In the circular area, Hipparcos tabulated parallaxes for only five stars (actually four, since $\sigma$ Ori D was given the parallax of $\sigma$ Ori Aa,Ab,B). Because of occasional saturation and mixing of the signals of the three components (Schaefer et al. 2016), the new Hipparcos data reduction of
Table 1 Youth features of the 13 Mayrit/TGAS cluster member candidates.

\begin{tabular}{lll}
\hline \hline Mayrit & Youth feature & Reference $^{a}$ \\
\hline \multirow{2}{*}{182305} & OB spectral type & SC71 \\
& X-rays & Cab07, Cab10 \\
189303 & OB spectral type & SC71, Gue81 \\
& X-rays & Fra06, Cab07, Cab10 \\
$(459340)$ & H $\alpha$ & Ste86, DK88, Cab08 \\
& Debris disc & Cab08, Her14 \\
524060 & X-rays & Cab07, Cab10 \\
& Debris disc & TO91, Her07, Mit15 \\
$(717307)$ & X-rays? & Fra06 \\
& Li I? & Cab06 \\
783254 & Li I & Wo196 \\
& X-rays, flare & Fra06, Cab07, Cab09, Cab10 \\
863116 & Li I, H $\alpha$ & Alc96 \\
& X-rays, flare & Fra06, Cab09, Cab10 \\
960106 & OB spectral type & JB81 \\
& X-rays & Cab10 \\
& Phot. variability & Nor84 \\
1160190 & None & $\ldots$ \\
$(1227243)$ & None & $\ldots$ \\
1285339 & Li I, H $\alpha$ & Tor95, Roj08 \\
& Transition disc & GL90, OvL04, Her07, Wil13 \\
& X-rays & Cab07 \\
$(1456284)$ & X-rays? & LSC08 \\
$(1468100)$ & Fast rotation? & GC93 \\
\hline \multirow{2}{*}{ (28) }
\end{tabular}

a References. SC71: Schild \& Chaffee 1971; Gue81: Guetter 1981; JB81: Joncas \& Borra 1981; Nor84: North 1984; Ste86; Stephenson 1986; DK88: Downes \& Keyes 1988; GL90: García-Lario et al. 1990; TO91: Tovmasyan \& Oganesyan 1991; GC93: Gray \& Corbally 1993; Tor95: Torres et al. 1995; Alc96: Alcalá et al. 1996; Wo196: Wolk 1996; OvL04: Oliveira \& van Loon 2004; Fra06: Franciosini et al. 2006; Her07: Hernández et al. 2007; Cab08: Caballero et al. 2008; LSC08: López-Santiago \& Caballero 2008; Roj08: Rojas et al. 2008; Cab09: Caballero et al. 2009; Wil13: Williams et al. 2013; Her14: Hernández et al. 2014; Mit15: Mittal et al. 2015.

van Leeuven (2007) provided larger uncertainties and less reliable values for the parallax of $\sigma$ Ori Aa,Ab,B than the original reduction by Perryman et al. (1997), so I kept the original values for homogeneity (denoted by HIP1 in last column of Table A1 . Three of the four independent values clustered at around $\pi \approx 2.5-2.8$ mas $(d \approx 350-400 \mathrm{pc})$.

Of the 46 stars in Table A1 39 were listed in the Gaia first data release (DR1; Gaia Collaboration et al. 2016). Five of the seven missing stars are the brightest and most massive ones towards the cluster: $\sigma$ Ori Aa,Ab,B (unresolved), D, and E, HD 37699, and HD $294271\left(V_{T} \approx 3.8-7.9 \mathrm{mag}, \mathcal{M} \approx\right.$ 3.9-17 $M_{\odot}$ ). The other two stars, HD 37686 and TYC 4771934-1, have expected $G$ magnitudes of about 9.2 mag and $11 \mathrm{mag}$ and are less affected than other fainter Gaia DR1 stars by the bright emission nebula IC 434, against which 
the Horsehead Nebula is silhouetted. In general, the Gaia $G$-band mean magnitude smoothly interpolates the Tycho $B_{T}, V_{T}$ (Høg et al. 2000), 2MASS $J, H, K_{s}$ (Skrutskie et al. 2006), and AllWISE $W 1, W 2, W 3$, and $W 4$ (Cutri et al. 2014) magnitudes.

Twenty-four of the 39 bright Gaia DR1 stars in TableA1 are tabulated in TGAS and have new parallax determinations. As expected, there is no other TGAS star in the surveyed area. This list of 24 stars includes 13 of the 29 bright, resolved, young stars and candidates in the $\sigma$ Orionis cluster identified by Caballero (2007). The 13 stars have a Mayrit designation in the second column of Table A1, and a TGAS reference in the last one. The Mayrit catalogue tabulated over $300 \sigma$ Orionis members and member candidates identified in a 2MASS+DENIS search accompanied by an exhaustive literature compilation of youth features (Caballero 2008c).

The other 11 TGAS stars in the 30 arcmin-radius area are either fore- or background interlopers that contaminate photometric and astrometric searches in the cluster. The new TGAS data reinforce this classification, based on the stars position in colour-magnitude diagrammes, parallactic distances (from $193 \pm 30 \mathrm{pc}$ to $1900 \pm 1000 \mathrm{pc}$ ) or total proper motions (from 4.0 mas a $^{-1}$ to 44.3 mas a $^{-1}$ ), apart from their known spectra in some cases. The poorly investigated stars TYC 4770-873-1 and TYC 4770-1129-1 were photometric cluster member candidates in Caballero (2007), but later were not listed as candidate members in the Mayrit catalogue. The 11 stars seem to be late-F/early-G dwarfs and $\mathrm{K} / \mathrm{M}$ giants.

I investigated in detail the actual cluster membership of the 13 Mayrit/TGAS stars, all of which were expected to be members in the young $\sigma$ Orionis cluster. However, since the publication of the Mayrit catalogue by Caballero (2008c), there have been numerous works devoted to study stars in the cluster with a variety of techniques (optical spectroscopy, X-rays, proper motion, mid-infrared - Section 11, and the actual cluster membership of some stars should be revisited. Table 1 summarises my investigations (see references in the footnote).

As shown in Table A1 four of the 13 stars have parallaxes that deviate from the rest of measurements. Of them, three have questionable features of youth or none at all: $(i)$ Mayrit $(717307)=$ TYC $4771-950-1$ is an F7 star with very faint $\mathrm{X}$-ray emission found only by Franciosini et al. (2006) and very weak lithium absorption found by $\mathrm{Ca}-$ ballero (2006). Although the Li I $\lambda \lambda 6707.76,6707.91$ Å doublet starts to appear at the $\mathrm{F} / \mathrm{G}$ spectral-type boundary in dwarf stars, it could be mistaken with the Fe I $26707.43 \AA$ line at low spectral resolutions; (ii) Mayrit (1456284) = TYC 4770-1261-1 was detected only in one EPIC/XMMNewton camera and with a low maximum likelihood parameter, so López-Santiago \& Caballero (2008) already called its true emission into question; and (iii) Mayrit (1227243) = HD 294275 is an early A-type dwarf with any signpost of youth. The fourth star with deviating parallax is
Table 2 Heliocentric distances to $\sigma$ Orionis $^{a}$.

\begin{tabular}{|c|c|c|}
\hline$d[\mathrm{pc}]$ & Method & Reference \\
\hline 440 & Photometry & Garrison 1967 \\
\hline 400 & Dynamical parallax $^{b}$ & Heintz 1974 \\
\hline $370 \pm 50$ & Spectroscopy ${ }^{c}$ & Hunger et al. 1989 \\
\hline $352_{-168}^{+166}$ & Parallax & Perryman et al. 1997 \\
\hline $380_{-87}^{+136}$ & Parallax & Maíz-Apellániz et al. 2004 \\
\hline $389_{-24}^{+34}$ & Photometry & Mayne \& Naylor 2008 \\
\hline $444 \pm 20$ & Photometry $^{d}$ & Sherry et al. 2008 \\
\hline$\sim 385$ & Dynamic parallax $^{e}$ & Caballero 20008b \\
\hline $407 \pm 11$ & Photometry & Bell et al. 2013 \\
\hline 380 & Interferometry & Hummel et al. 2013 \\
\hline $381 \pm 7$ & Interferometry & Schaefer 2013 \\
\hline $387.5 \pm 1.3$ & Interferometry & Schaefer et al. 2016 \\
\hline $360_{-35}^{+44}$ & Parallax $^{f}$ & This work \\
\hline
\end{tabular}

a There are other determinations of the distance to the Ori OB1b group by Brown et al. (1994), de Zeeuw et al. (1999), and Hernández et al. (2005). The parallactic determination by van Leeuwen (2007), with a large uncertainty, is not tabulated either.

$b$ Under the double $\sigma$ Ori AB scenario.

$c$ From a $\sigma$ Ori D spectrogram.

$d$ For solar metallicity (González-Hernández et al. 2008).

$e$ Under the triple $\sigma$ Ori Aa,Ab,B scenario.

$f$ Based on eight TGAS stars.

Mayrit (459340) $=$ StHA 50, an A2-6 V star with a powerful $\mathrm{H} \alpha$ emission (Caballero et al. 2008) and a debris/evolved disc (Hernández et al. 2014). Instead of being a UX Ori-type star with a scattering edge-on disc in $\sigma$ Orionis, as proposed by Caballero et al. (2008), it could be an unusual, isolated, Herbig Ae/Be star in the background, at about $300 \mathrm{pc}$ below the Galactic plane. The Mayrit designations of the four stars are written in parenthesis in Tables 1 and $\mathrm{A1}$ and this paragraph for showing that they should not be used any more, as the stars are not actual $\sigma$ Orionis members. A fifth star, also with a Mayrit designation in parenthesis and with only one controvertible signpost of youth ("fast rotation?" - Table 1), namely Mayrit (1468100) = HD 294301, has an abnormally large total proper motion of 9.66 mas a $^{-1}$. It could be either a young intermediate-mass star in the process of ejection via dynamical interactions within the Ori OB1b association or, more likely, an old spectroscopic-binary interloper.

The other eight TGAS star 1 towards the $\sigma$ Orionis cluster have conclusive youth features (OB spectral types, circumstellar discs, lithium in absorption, $\mathrm{H} \alpha$ and/or X-rays in strong emission, flaring activity) and parallaxes that vary within a narrow range from $2.43 \pm 0.27$ mas (Mayrit 783254) to $3.24 \pm 0.52$ mas (Mayrit 863116 ). The mean parallax and standard deviation of the eight TGAS stars are $\operatorname{mean}_{8}(\pi)=$

\footnotetext{
1 According to Kharchenko et al. (2004), four of the eight Mayrit/TGAS stars also had kinematic and photometric probabilities consistent with cluster membership at $2 \sigma\left(p_{\text {kin }}, p_{\mathrm{ph}}>0.14\right)$.
} 
2.78 mas and $\operatorname{std}_{8}(\pi)=0.22$ mas, from which one can estimate a robust heliocentric distance.

However, alternative parallax values can be derived. On the one hand, if the two most extreme cases (Mayrit 783254 and Mayrit 863116) are discarded, the mean parallax with six stars remains very similar but the standard deviations gets lower, with $\operatorname{mean}_{6}(\pi)=2.77$ mas and $\operatorname{std}_{6}(\pi)=$ 0.06 mas. On the other case, if three of the four independent HIP1 parallaxes are added to the set (it is not clear whether HD 37699 actually belongs to $\sigma$ Orionis, or to a new young population in the background, as StHA 50; Caballero 2007), the mean and standard deviation of the parallax with 11 TGAS+HIP1 stars are much the same: $\operatorname{mean}_{11}(\pi)=2.74$ mas and $\operatorname{std}_{11}(\pi)=0.21$ mas. Weighted means, medians, Bayesian statistics, or even Lutz-Kelker corrections will be worth investigating only when the second Gaia data release is available in April 2018.

Following the comment on correlated systematic terms and cluster mean parallaxes by Gaia Collaboration et al. (2016), by which a systematic component of $\sim 0.3$ mas should be added to the parallax uncertainties, the best estimate that I can make with TGAS data for the $\sigma$ Orionis cluster is $2.78 \pm 0.30$ mas, corresponding to a distance of $360_{-35}^{+44} \mathrm{pc}$.

\section{Discussion and conclusions}

The derived TGAS distance to $\sigma$ Orionis of $d=360_{-35}^{+44} \mathrm{pc}$ fits in the lower end of all previous determinations, as comprehensively shown in Table 2. In particular, the TGAS distance is equal within uncertainties to the most precise and accurate distance to $\sigma$ Orionis to date, by Schaefer et al. (2016), who used an exhaustive set of interferometric and radial-velocity data. We will have to wait for Gaia DR2 for a proper comparison and identification of systematic differences between both astrometric (from space) and interferometric+radial-velocity (from the ground) distances. In any case, this work paves the way for such a future comparison.

The TGAS distance resembles the values used by Zapatero-Osorio et al. (2000), Béjar et al. (1999, 2001), Lodieu et al. (2009), and Peña-Ramírez et al. (2012) from Perryman et al. (1997; $d=352_{-168}^{+166}$ ), and Caballero et al. (2007) and Bihain et al. (2009) from Brown et al. (1994; $d$ $=360_{-60}^{+70}$ ), which are the most representative works on the bottom of the initial mass function in the cluster. Therefore, their results are not affected by the new TGAS distance (and only slightly by Schaefer et al. 2016's). However, the deprecated values of 400-450 pc have been used in the last years by many teams worldwide for a variety of science topics, which may affect their results (e.g., Cody \& Hillenbrand 2011 for brown-dwarf variability; Williams et al. 2013 for protoplanetary discs; Townsend et al. 2013 and Oksala et al. 2015 for the peculiar star $\sigma$ Ori E; Koenig et al. 2015 for deep infrared surveys).
The mean proper motion of the eight Mayrit/TGAS cluster members is $\left(\mu_{\alpha} \cos \delta, \mu_{\delta}\right)=(-0.2 \pm 1.5$, $+0.0 \pm 0.8)$ mas a $^{-1}$, which is consistent with previous determinations by Kharchenko et al. (2005) and Caballero (2007, 2010). This low mean proper motion, due to the location of $\sigma$ Orionis in the antapex and at almost $400 \mathrm{pc}$, will not prevent that after further Gaia data releases the internal kinematic dispersion of the open cluster is investigated in a per-star basis (at the end of the Gaia mission, some $\sigma$ Orionis stars will have proper motion uncertainties of only a few tens $\mu$ as $\mathrm{a}^{-1}$ ). Proper-motion analysis has been already used for discarding photometric cluster member candidates in the cluster (Kharchenko et al. 2004; Caballero 2007, 2010; Lodieu et al. 2009), and it seems to be the most efficient rejecting method for A- and F-type stars, which signposts of youth are not as evident as in other spectral types (such as the Li I absorption doublet in fainter, very young GKM-type stars and brown dwarfs).

Although the derived TGAS distance to $\sigma$ Orionis supersedes most previous determinations and is consistent with the value at $387.5 \pm 1.3$ pc of Schaefer et al. (2016), which should be used as a reference until the analyses of Gaia DR2 data are made public, perhaps the most striking result in this work was obtained in the process of selection of bona fide cluster members. In this process, I discarded five Mayrit stars from the initial list of targets (including the Herbig Ae/Be star StHA 50), which represent more than half of all previously considered cluster member candidates with masses between 1.2 $M_{\odot}$ and $2.1 M_{\odot}$ (Caballero 2007). In other words, there could be more B stars in $\sigma$ Orionis than $\mathrm{A}$ and $\mathrm{F}$ stars together. Besides, the addition of a third massive star in the central astrometric "binary", $\sigma$ Ori Ab $\left(\mathcal{M} \sim 12.8 M_{\odot}-\right.$ Simón-Díaz et al. 2015; Schaefer et al. 2016), leads to a flattening of the slope of the mass spectrum with respect to previous determinations at the highmass end (Caballero 2007; Peña-Ramírez et al. 2012). The slope value can be at about $\alpha(\equiv-\gamma \equiv 1-\Gamma) \approx+1.5$ in the stellar mass interval from 1.2 to $17 M_{\odot}$, well below the Salpeter's one at +2.35 . A careful determination of the actual slopes of the $\sigma$ Orionis mass spectrum with only Tycho2 data (using roughly the same star sample as is in this work with TGAS data) was already shown to be inconclusive by Caballero (2011), while the use of the full Gaia DR1 dataset is out of the scope of this work.

With the same methodology as the one presented here, and when new precise parallaxes, proper motions, and $B_{P}$ and $R_{P}$ photometry from Gaia DR2 are available for a much larger number of stars, it will be possible to go one step ahead, and create a clean list of bona fide $\sigma$ Orionis members from the high-mass end down to the star/brown boundary, which will help measuring more accurate cluster mass function indices, isochronal ages, and disc and activity fractions.

Acknowledgements. JAC thanks the referee, R.-D. Scholz, for his constructive input. Financial support was provided by the Klaus Tschira Stiftung and Spanish Ministerio de 
Economía y Competitividad under grants AYA2014-54348C3-2-R and AYA2015-74151-JIN. This research made use of the SIMBAD, operated at Centre de Données astronomiques de Strasbourg, France, the NASA's Astrophysics Data System, and of data from the European Space Agency (ESA) mission Gaia (http://www.cosmos.esa.int/gaia), processed by the Gaia Data Processing and Analysis Consortium (DPAC, http://www . cosmos. esa.int/web/gaia/dpac/consortium). Funding for the DPAC has been provided by national institutions, in particular the institutions participating in the Gaia Multilateral Agreement.

\section{References}

Alcalá, J. M., Terranegra, L., Wichmann, R. et al. 1996, A\&AS, 119,7

Andrews, S. M., Reipurth, B., Bally, J., \& Heathcote, S. R. 2004, ApJ, 606, 353

Béjar, V. J. S., Zapatero Osorio, M. R., Rebolo, R. et al. 1999, ApJ, 521,671

Béjar, V. J. S., Martín, E. L., Zapatero Osorio, M. R. et al. 2001, ApJ, 556, 830

Béjar, V. J. S., Zapatero Osorio, M. R., Rebolo, R. et al. 2011, ApJ, 743,64

Bell, C. P. M., Naylor, T., Mayne, N. J. et al. 2013, MNRAS, 434, 806

Bihain, G., Rebolo, R., Zapatero Osorio, M. R. et al. 2009, A\&A, 506, 1169

Brown, A. G. A., de Geus, E. J., \& de Zeeuw, P. T. 1994, A\&A, 289,101

Caballero, J. A. 2006, PhD thesis, Universidad de La Laguna, Tenerife, Spain

Caballero, J. A. 2007, A\&A, 466, 917

Caballero, J. A. 2008a, MNRAS, 383, 375

Caballero, J. A. 2008b, MNRAS, 383, 750

Caballero, J. A. 2008c, A\&A, 487, 667

Caballero, J. A. 2010, A\&A, 514, A18

Caballero, J. A. 2011, Stellar Clusters \& Associations: a RIA Workshop on Gaia, 108

Caballero, J. A. 2014, The Observatory, 134, 273

Caballero, J. A. \& Solano, E. 2008, A\&A, 485, 931

Caballero, J. A., Béjar, V. J. S., Rebolo, R., \& Zapatero Osorio, M. R. 2004, A\&A, 424, 857

Caballero, J. A., Martín, E. L., Zapatero Osorio, M. R. et al. 2006, A\&A, 445, 143

Caballero, J. A., Béjar, V. J. S., Rebolo, R. et al. 2007, A\&A, 470, 903

Caballero, J. A., Valdivielso, L., Martín, E. L. et al. 2008, A\&A, 491,515

Caballero, J. A., López-Santiago, J., de Castro, E., \& Cornide, M. 2009, AJ, 137, 5012

Caballero, J. A., Albacete-Colombo, J. F., \& López-Santiago, J. 2010, A\&A, 521A, 45

Caballero, J. A., Cabrera-Lavers, A., García-Álvarez, D., \& Pascual, S. 2012, A\&A, 546, A59

Caballero, J. A., Bouy, H., \& Lillo-Box, J. 2016, The Observatory, 136,226

Cody, A. M. \& Hillenbrand, L. A. 2010, ApJS, 191, 389

Cody, A. M. \& Hillenbrand, L. A. 2011, ApJ, 741, 9

Cutri, R. M. et al. 2014, VizieR on-line data catalogue II/328

de Zeeuw, P. T., Hoogerwerf, R., de Bruijne, J. H. J. et al. 1999, AJ, 117, 354
Downes, R. A. \& Keyes, C. D. 1988, AJ, 96, 777

Franciosini, E., Pallavicini, R., \& Sanz-Forcada, J. 2006, A\&A, 446,501

Gaia Collaboration, Brown, A. G. A., Vallenari, A. et al. 2016, A\&A, 595, A2

García-Lario, P., Manchado, A., Suso, S. R. et al. 1990, A\&AS, 82,497

Garrison, R. F. 1967, PASP, 79, 433

Gatti, T., Natta, A., Randich, S. et al. 2008, A\&A, 481, 423

Goicoechea, J. R., Pety, J., Gerin, M. et al. 2006, A\&A, 456, 565

González Hernández, J. I., Caballero, J. A., Rebolo, R. et al. 2008, A\&A, 490, 1135

Gray, R. O. \& Corbally, C. J. 1993, AJ, 106, 632

Groote, D. \& Hunger, K. 1982, A\&A, 116, 64

Guetter, H. H. 1981, AJ, 86, 1057

Haro, G. \& Moreno, A. 1953, Boletín de los Observatorios de Tonantzintla y Tacubaya, 1,11

Hartkopf, W. I., Mason, B. D., \& McAlister, H. A. 1996, AJ, 111, 370

Heintz, W. D. 1974, AJ, 79, 819

Hernández, J., Calvet, N., Hartmann, L. et al. 2005, AJ, 129, 856

Hernández, J., Hartmann, L., Megeath, T. et al. 2007, ApJ, 662, 1067

Hernández, J., Calvet, N., Pérez, A. et al. 2014, ApJ, 794, 36

Høg, E., Kuzmin, A., Bastian, U. et al. 1998, A\&A, 335, L65

Høg, E., Fabricius, C., Makarov, V. V. et al. 2000, A\&A, 355, L27

Hummel, C. A., Zavala, R. T., \& Sanborn, J. 2013, Central European Astrophysical Bulletin, 37, 127

Hunger, K., Heber, U., \& Groote, D. 1989, A\&A, 224, 57

Joncas, G. \& Borra, E. F. 1981, A\&A, 94, 134

Kenyon, M. J., Jeffries, R. D., Naylor, T. et al. 2005, MNRAS, 356, 89

Kharchenko, N. V., Piskunov, A. E., Röser, S. et al. 2004, AN, 325, 740

Kharchenko, N. V., Piskunov, A. E., Röser, S. et al. 2005, A\&A, 438,1163

Koenig, X., Hillenbrand, L. A., Padgett, D. L., \& DeFelippis, D. 2015, AJ, 150, 100

Kubiak, K., Alves, J., Bouy, H. et al. 2016, A\&A, in press (eprint arXiv:1609.04948)

Lodieu, N., Zapatero Osorio, M. R., Rebolo, R. et al. 2009, A\&A, 505,1115

López-Santiago, J. \& Caballero, J. A. 2008, A\&A, 491, 961

Luhman, K. L., Hernández, J., Downes, J. J., Hartmann, L., \& Briceño, C. 2008, ApJ, 688, 362

Maíz-Apellániz, J., Walborn, N. R. et al. 2004, ApJS, 151, 103

Mason, B. D., Gies, D. R., Hartkopf, W. I. et al. 1998, AJ, 115, 812

Maucó, K., Hernández, J., Calvet, N. et al. 2016, ApJ, 829, 38

Mayne, N. J. \& Naylor, T. 2008, MNRAS, 386, 261

Mittal, T., Chen, C. H., Jang-Condell, H. et al. 2015, ApJ, 798, 87

Mokler, F. \& Stelzer, B. 2002, A\&A, 391, 1025

North, P. 1984, A\&AS, 55, 259

Oksala, M. E., Wade, G. A., Townsend, R. H. D. et al. 2012, MNRAS, 419, 959

Oksala, M. E., Kochukhov, O., Krtička, J. et al. 2015, MNRAS, 451,2015

Oliveira, J. M. \& van Loon, J. Th. 2004, A\&A, 418, 663

Oliveira, J. M., Jeffries, R. D. \& van Loon, J. Th. 2004, MNRAS, 347,1327

Peña-Ramírez, K., Béjar, V. J. S., Zapatero Osorio, M. R. et al. 2012, ApJ, 754, 30

Perryman, M. A. C., Lindegren, L., Kovalevsky, J. et al. 1997, A\&A, 323, L49 
Pety, J., Teyssier, D., Fossé, D. et al. 2005, A\&A, 435, 885

Pety, J., Gratier, P., Guzmán, V. et al. 2012, A\&A, 548, A68

Pound, M. W., Reipurth, B., \& Bally, J. 2003, AJ, 125, 2108

Reipurth, B., Bally, J., Fesen, R. A., \& Devine, D. 1998, Nature, 396,343

Riaz, B., Thompson, M., Whelan, E. T., \& Lodieu, N. 2015, MNRAS, 446, 2550

Rigliaco, E., Natta, A., Randich, S. et al. 2011a, A\&A, 525, A47

Rigliaco, E., Natta, A., Randich, S. et al. 2011b, A\&A, 526, L6

Rigliaco, E., Natta, A., Testi, L. et al. 2012, A\&A, 548, A56

Rojas, G., Gregorio-Hetem, J., \& Hetem, A. 2008, MNRAS, 387, 1335

Sacco, G. G., Franciosini, E., Randich, S., \& Pallavicini, R. 2008, A\&A, 488, 167

Schaefer, G. H. 2013, EAS, 64, 181

Schaefer, G. H., Hummel, C. A., Gies, D. R. et al. 2016, AJ, 152, 213

Schild, R. E. \& Chaffee, F. 1971, ApJ, 169, 529

Scholz, A. \& Eislöffel, J. 2004, A\&A, 419, 249

Scholz, A., Xu, X., Jayawardhana, R. et al. 2009, ApJ, 702, 805

Sherry, W. H., Walter, F. M., \& Wolk, S. J. 2004, AJ, 128, 2316

Sherry, W. H., Walter, F. M., Wolk, S. J., \& Adams, N. R. 2008, AJ, 135, 1616

Simón-Díaz, S., Caballero, J. A., \& Lorenzo, J. 2011, ApJ, 742, 55

Simón-Díaz, S., Caballero, J. A., Lorenzo, J. et al. 2015, ApJ, 799, 169

Skinner, S. L., Sokal, K. R., Cohen, D. H. et al. 2008, ApJ, 683, 796

Skrutskie, M. F., Cutri, R. M., Stiening, R. et al. 2006, AJ, 131, 1163

Stephenson, C. B. 1986, ApJ, 300, 779

Torres, C. A. O., Quast, G., de La Reza, R. et al. 1995, AJ, 109, 2146

Tovmasyan, G. M. \& Oganesyan, R. Kh. 1991, Astrofizika, 34, 301

Townsend, R. H. D., Owocki, S. P., \& Groote, D. 2005, ApJ, 630, L81

Townsend, R. H. D., Oksala, M. E., Cohen, D. H. et al. 2010, ApJ, 714, L318

Townsend, R. H. D., Rivinius, Th., Rowe, J. F. et al. 2013, ApJ, 769,33

van Leeuwen, F. 2007, A\&A, 474, 653

Walborn, N. R. 1974, ApJ, 191, L95

Walter, F. M., Sherry, W. H., Wolk, S. J., \& Adams, N. R. 2008 , Handbook of Star Forming Regions, Volume I: The Northern Sky ASP Monograph Publications, Vol. 4. Edited by Bo Reipurth, p. 732

Williams, J. P., Cieza, L. A., Andrews, S. M. et al. 2013, MNRAS, 435,1671

Wolk, S. J. 1996, Ph.D. thesis, State University of New York, NY, USA

Zacharias, N., Monet, D. G., Levine, S. E. et al. 2004, AAS, 205, 4815

Zapatero Osorio, M. R., Béjar, V. J. S., Martín, E. L. et al. 2000, Science, 290, 103

Zapatero Osorio, M. R., Béjar, V. J. S., Pavlenko, Ya. et al. 2002, A\&A, 384, 937

Zapatero Osorio, M. R., Caballero, J. A., Béjar et al. 2007, A\&A, 472, L9

\section{A Table A1}


Table A1 Relevant astro-photometric data of the brightest stars in the $\sigma$ Orionis cluster $^{a}$.

\begin{tabular}{|c|c|c|c|c|c|c|c|c|}
\hline Name & Mayrit & $\begin{array}{l}\text { Sp. } \\
\text { type }\end{array}$ & $\begin{array}{c}V_{T} \\
{[\mathrm{mag}]}\end{array}$ & $\begin{array}{c}G \\
{[\mathrm{mag}]}\end{array}$ & $\begin{array}{c}J \\
{[\mathrm{mag}]}\end{array}$ & $\begin{array}{c}\mu \\
{\left[\mathrm{mas} \mathrm{a}^{-1}\right]}\end{array}$ & $\begin{array}{c}\pi \\
{[\mathrm{mas}]}\end{array}$ & Ref. \\
\hline$\sigma$ Ori Aa,Ab,B & $\mathrm{AB}$ & $09.5 \mathrm{~V}+$ & 3.763 & $\ldots$ & 4.752 & 4.63 & $2.84 \pm 0.91$ & HIP1 \\
\hline$\sigma$ Ori $\mathrm{C}$ & 11238 & $\mathrm{~A} 2 \mathrm{~V}$ & $9.43^{b}$ & 9.450 & 9.086 & 0.00 & $\ldots$ & NOMAD \\
\hline$\sigma$ Ori D & 13084 & B2 V & 6.557 & $\ldots$ & 7.116 & 4.62 & $(2.84 \pm 0.91)$ & HIP1 \\
\hline$\sigma$ Ori E & 42062 & $\mathrm{~B} 2 \mathrm{Vp}$ & 6.344 & $\ldots$ & 6.974 & 2.61 & $\ldots$ & TYC1 \\
\hline HD 294272 A & $182305^{c}$ & B9.5 III & 8.389 & 8.464 & 8.346 & 2.07 & $2.75 \pm 0.34$ & TGAS \\
\hline HD 294272 B & $189303^{c}$ & B8 V & 8.554 & 8.766 & 8.779 & 0.67 & $2.75 \pm 0.42$ & TGAS \\
\hline HD 294271 & 208324 & B5 V & 7.856 & $\ldots$ & 8.100 & 0.72 & $\ldots$ & TYC2 \\
\hline HD $37525 \mathrm{AB}$ & 306125 & B5 Vp & 8.058 & 8.088 & 8.131 & 2.14 & $2.55 \pm 0.99$ & HIP1 \\
\hline StHA 50 & $(459340)$ & A2-6 Ve & 11.275 & 11.180 & 10.666 & 4.25 & $0.76 \pm 0.31$ & TGAS \\
\hline HD 294273 & 521210 & A3 & 10.828 & 10.606 & 10.176 & 4.64 & $\ldots$ & TYC1 \\
\hline HD 37564 & $524060^{c}$ & A8: V & 8.443 & 8.427 & 7.976 & 0.35 & $2.85 \pm 0.36$ & TGAS \\
\hline TYC 4771-950-1 & (717307) & F7 & 11.429 & 11.084 & 10.088 & 14.62 & $1.90 \pm 0.27$ & TGAS \\
\hline TYC 4771-661-1 & $\ldots$ & K-M: & 11.899 & 11.641 & 10.617 & 26.07 & $\ldots$ & TYC2 \\
\hline TYC 4771-720-1 & $\ldots$ & K-M: & 11.787 & 11.331 & 10.269 & 26.07 & $\ldots$ & TYC2 \\
\hline 2E 1455 & $783254^{c}$ & $\ldots$ & 11.015 & 10.670 & 9.255 & 0.56 & $2.43 \pm 0.27$ & TGAS \\
\hline HD 294278 & $\ldots$ & $\mathrm{K} 2$ & 9.941 & 9.310 & 7.592 & 8.54 & $2.25 \pm 0.28$ & TGAS \\
\hline HD $294300 \mathrm{AB}$ & $863116^{c}$ & G5-K0 & 10.195 & 9.657 & 8.462 & 0.88 & $3.24 \pm 0.52$ & TGAS \\
\hline HD 294269 & $\ldots$ & G0 & 10.795 & 10.379 & 9.178 & 44.28 & $4.12 \pm 0.23$ & TGAS \\
\hline V1147 Ori & $960106^{c}$ & B9 IIIp & 8.992 & 9.026 & 8.876 & 1.97 & $2.77 \pm 0.35$ & TGAS \\
\hline TYC 4771-962-1 & 968292 & $\ldots$ & 11.101 & 11.000 & 10.202 & 3.89 & $\ldots$ & TYC2 \\
\hline TYC 4771-934-1 & $\ldots$ & K-M: & 12.870 & $\ldots$ & 8.948 & 7.97 & $\ldots$ & TYC2 \\
\hline HD 294274 & $\ldots$ & G0 & 10.758 & 10.497 & 9.428 & 21.39 & $3.06 \pm 0.25$ & TGAS \\
\hline TYC 4771-621-1 & $\ldots$ & K-M: & 10.920 & 10.642 & 9.796 & 13.12 & $\ldots$ & TYC2 \\
\hline TYC 4771-873-1 & $\ldots$ & F7-9 & 12.031 & 12.097 & 11.125 & 6.12 & $1.22 \pm 0.57$ & TGAS \\
\hline HD 37333 & 1116300 & A1 Va & 8.508 & 8.529 & 8.413 & 5.11 & $2.52 \pm 1.08$ & HIP1 \\
\hline HD 294279 & $1160190^{c}$ & F3-5 & 10.685 & 10.587 & 9.873 & 3.06 & $2.80 \pm 0.25$ & TGAS \\
\hline TYC 4771-1468-1 & $\ldots$ & $\mathrm{K}-\mathrm{M}:$ & 11.136 & 10.466 & 8.616 & 5.73 & $0.53 \pm 0.27$ & TGAS \\
\hline HD 294275 & (1227243) & A $1 \mathrm{~V}$ & 9.431 & 9.419 & 9.256 & 3.50 & $3.53 \pm 0.36$ & TGAS \\
\hline HD 294277 & $\ldots$ & $\mathrm{K} 2$ & 9.737 & 8.810 & 6.773 & 8.24 & $1.57 \pm 0.30$ & TGAS \\
\hline HD 294268 & $1285339^{c}$ & F5 & 10.507 & 10.262 & 9.393 & 1.01 & $2.68 \pm 0.47$ & TGAS \\
\hline HD 37545 & 1288163 & B9 V & 9.277 & 9.306 & 9.261 & 2.20 & $\ldots$ & TYC2 \\
\hline HD 294270 & $\ldots$ & G0 & 10.960 & 10.665 & 9.780 & 28.81 & $\ldots$ & TYC2 \\
\hline TYC 4770-924-1 & $\ldots$ & K-M: & 11.938 & 11.947 & 10.725 & 36.08 & $5.19 \pm 0.82$ & TGAS \\
\hline HD 37686 & 1359077 & B9.5 Vn & 9.186 & $\ldots$ & 9.207 & 2.97 & $\ldots$ & TYC2 \\
\hline HD 294298 & 1366055 & G0: & 10.975 & 10.608 & 9.339 & 1.84 & $\ldots$ & TYC2 \\
\hline TYC 4770-1432-1 & $\ldots$ & K-M: & 12.337 & 12.158 & 11.077 & 30.90 & $\ldots$ & TYC2 \\
\hline TYC 4770-1261-1 & (1456284) & $\ldots$ & 11.498 & 11.686 & 10.721 & 9.53 & $3.94 \pm 0.85$ & TGAS \\
\hline HD 294301 & 1468100 & F2 V(n) & 11.139 & 10.905 & 10.210 & 9.66 & $2.64 \pm 0.24$ & TGAS \\
\hline HD 294276 & $\ldots$ & G0 & 10.418 & 10.203 & 9.183 & 62.32 & $\ldots$ & TYC1 \\
\hline HD 37699 & 1548068 & B5 Vn & 7.590 & $\ldots$ & 7.841 & 0.95 & $0.63 \pm 1.01$ & HIP1 \\
\hline TYC 4771-1012-1 & $\ldots$ & K-M: & 11.263 & 10.865 & 8.998 & 9.74 & $\ldots$ & TYC2 \\
\hline HD 294297 & $\ldots$ & F6-8 & 10.193 & 9.925 & 9.062 & 30.59 & $\ldots$ & TYC1 \\
\hline HD 294307 & $\ldots$ & F8 & 10.552 & 10.256 & 9.439 & 21.61 & $4.38 \pm 0.28$ & TGAS \\
\hline TYC 4770-1129-1 & $\ldots$ & $\ldots$ & 11.827 & 12.171 & 11.232 & 5.67 & $0.91 \pm 0.38$ & TGAS \\
\hline HD 294280 & $\ldots$ & K5 & 9.865 & 8.774 & 6.429 & 4.03 & $1.68 \pm 0.33$ & TGAS \\
\hline TYC 4770-1018-1 & $\ldots$ & K-M: & 11.224 & 10.426 & 8.620 & 5.02 & $0.93 \pm 0.25$ & TGAS \\
\hline
\end{tabular}

a Star name, Mayrit designation, spectral type, Tycho-2 $V_{T}$, Gaia $G$, and 2MASS $J$ magnitudes, total proper motion, parallax, and reference for the last two parameters. Stars are sorted by angular separation to $\sigma$ Ori Aa,Ab,B. An extended version of this table, with coordinates, Tycho-2, and TGAS proper motions, and magnitudes from $B_{T}$ to W4, is (WILL BE) available online via VizieR.

${ }^{b} V_{T}$ magnitude of $\sigma$ Ori C derived from $V$ in Sherry et al. (2008).

$c$ The eight Mayrit/TGAS stars used for the parallax determination. 